\title{
$k$-Congruences and the Zariski topology in semirings
}

\author{
Song-Chol Han (iD) \\ Faculty of Mathematics, Kim Il Sung University, Pyongyang, Democratic People's Republic of Korea
}

\begin{abstract}
The purpose of this paper is to study topological properties of both the set of all $k$-prime ideals and the set of all $k$-prime congruences for any commutative semiring with zero and identity. We first prove that the $k$-prime spectrum, i.e. the set of all $k$-prime ideals equipped with the Zariski topology is a spectral space, and then prove that the set of all $k$-prime congruences is homeomorphic to the $k$-prime spectrum with respect to their Zariski topologies.
\end{abstract}

Mathematics Subject Classification (2020). 16Y60, 54F65, 13A15

Keywords. semiring, ideal, congruence, prime ideal, Zariski topology

\section{Introduction}

The notion of semirings was introduced by Vandiver [17] in 1934. Semirings are algebraic systems that generalize both rings and distributive lattices and have many applications in diverse branches of mathematics and computer science $[5,7,13]$. Semirings have two binary operations of addition and multiplication which are connected by the ring-like distributive laws. But unlike in rings, subtraction is not allowed in semirings that are not rings. For this reason, there are considerable differences between ring theory and semiring theory and many results in ring theory have no analogues in semirings. In order to narrow the gap, Henriksen [8] in 1958 defined $k$-ideals in semirings. From the fact that $k$-ideals coincide with ring ideals in any ring, $k$-ideals in semirings have been a very interesting subject of research $[6,12,15,18,19]$.

The objective of the present paper is to study topological properties of both the set of all $k$-prime ideals with the Zariski topology and the set of all $k$-prime congruences with the Zariski topology for any commutative semiring with zero and identity. For this purpose, we first investigate topological properties of the $k$-prime spectrum, i.e. the set of all $k$-prime ideals equipped with the Zariski topology for any commutative semiring with zero and identity, and then prove that the $k$-prime spectrum and the set of all $k$-prime congruences are homeomorphic with respect to their Zariski topologies.

It is well known that the prime spectrum of a commutative ring with identity plays an important role in commutative algebra, algebraic geometry and lattice theory $[2,3]$.

Email address: sc.han@ryongnamsan.edu.kp

Received: 03.09.2019; Accepted: 21.10.2020 
For any commutative semiring with zero and identity, Golan [5] proved that the prime spectrum, i.e. the set of all prime ideals with the Zariski topology is a compact $T_{0}$-space, and Pena, Ruza and Vielma [14] proved that the prime spectrum is a spectral space.

It is shown below that the $k$-prime spectrum is a subspace of the prime spectrum for any commutative semiring with zero and identity. However, it has not been known even whether the $k$-prime spectrum is compact for any commutative semiring with zero and identity. In previous researches, Atani and Atani [1] considered topological properties of the prime spectrum of a commutative $k$-semiring, i.e. a commutative semiring with zero and identity whose ideals are all $k$-ideals. But one can see that this is a special case where the $k$-prime spectrum coincides with the prime spectrum. And Lescot [11] proved that the set of all saturated prime ideals with the Zariski topology is a spectral space for a characteristic one semiring. Note that a characteristic one semiring is an additively idempotent commutative semiring with zero and identity and saturated ideals are just $k$ ideals. Summing up, topological properties of the $k$-prime spectra have not been studied much for general commutative semirings with zero and identity.

The rest of this paper is as follows. Section 2 is for preliminaries. In Section 3, for any semiring, we define a $k$-congruence and show that $k$-congruences are in a one-toone correspondence with $k$-ideals. In Section 4 , for any commutative semiring with zero and identity, we study topological properties of the $k$-prime spectrum in detail and thus prove that the $k$-prime spectrum is a spectral space. In Section 5 , for any commutative semiring with zero and identity, we introduce the Zariski topology on the set of all $k$ prime congruences and prove that the resulting topological space is homeomorphic to the $k$-prime spectrum.

\section{Preliminaries}

In this section, we recall some known definitions and facts [4-7].

Throughout this paper, $R$ denotes a semiring $(R,+, \cdot)$, unless otherwise stated. That is to say, $(R,+)$ is a commutative semigroup, $(R, \cdot)$ is a semigroup, and multiplication distributes over addition from either side. If $R$ has an additively neutral element 0 and $0 r=r 0=0$ for all $r \in R$, then 0 is called a zero of $R$. We write $0 \in R$ when $R$ has a zero 0 . If $R$ has a multiplicatively neutral element 1 , then 1 is called an identity of $R$. $R$ is called a commutative semiring if multiplication is commutative. Below, we assume that $|R| \geqslant 2$ to avoid trivial exceptions.

A nonempty subset $A$ of $R$ is called an ideal of $R$ if $a+b \in A$ and $r a$, ar $\in A$ for all $a, b \in A$ and $r \in R$. An ideal $A$ of $R$ is said to be proper if $A \neq R$. $R$ and $\{0\}$ (if $0 \in R$ ) are said to be trivial ideals of $R$. Denote by $\mathcal{J}(R)$ the family of all ideals of $R$. For an ideal $A$ of $R$, the set

$$
\bar{A}=\{x \in R \mid x+a=b \text { for some } a, b \in A\}
$$

is called the subtractive closure or $k$-closure of $A$ in $R$. Then $\bar{A}$ is an ideal of $R$ and it holds that $A \subseteq \bar{A}$ and $\overline{(\bar{A})}=\bar{A}$. $A$ is said to be $k$-closed in $R$ if $A=\bar{A}$. For ideals $A$ and $B$ of $R, A \subseteq B$ implies $\bar{A} \subseteq \bar{B}$. An ideal $A$ of $R$ is called a subtractive ideal or $k$-ideal of $R$ if $A=\bar{A}$. If $A$ is an ideal of $R$, then $\bar{A}$ is a $k$-ideal of $R$. $R$ is a $k$-ideal of itself, and $\{0\}$ is also a $k$-ideal of $R$ if $0 \in R$. Denote by $\mathcal{K J}(R)$ the family of all $k$-ideals of $R$.

An equivalence relation $\equiv$ on $R$ is called a congruence on $R$ if for any $a, b, c \in R, a \equiv b$ implies $a+c \equiv b+c, a c \equiv b c$ and $c a \equiv c b$. The family of all congruences on $R$ is denoted by $\mathrm{C}(R)$. Given a congruence $\theta$ on $R$, the quotient set $R / \theta=\{[x] \mid x \in R\}$ consisting of all the congruence classes forms a semiring under the operations defined as $[x]+[y]=[x+y]$ and $[x] \cdot[y]=[x y]$ for $x, y \in R$. The semiring $R / \theta$ is called the quotient semiring of $R$ by $\theta$. If $0 \in R$, then [0] is a zero of $R / \theta$.

An ideal $A$ of $R$ defines a congruence $\kappa_{A}$ on $R$ by

$$
x \kappa_{A} y \Longleftrightarrow x+a=y+b \text { for some } a, b \in A \text {. }
$$


Then the $k$-closure $\bar{A}$ of $A$ is a zero of the quotient semiring $R / \kappa_{A}$. In addition, $\kappa_{A}=\kappa_{\bar{A}}$.

A $k$-semiring is a semiring whose ideals are all $k$-ideals [1].

A proper $k$-ideal $A$ of a semiring $R$ is said to be $k$-maximal in $R$ if for each $k$-ideal $B$ of $R, A \subset B \subseteq R$ implies $B=R[15,18]$. Denote by $\operatorname{Max}_{k}(R)$ the family of all $k$-maximal ideals of $R$.

Lemma 2.1 ([15,18]). If $R$ is a semiring with zero and identity, then each proper $k$-ideal of $R$ is contained in a k-maximal ideal of $R$.

A proper ideal $A$ of a commutative semiring $R$ is said to be prime if $x y \in A$ with $x, y \in R$ implies $x \in A$ or $y \in A$ [15]. The family of all prime ideals of $R$ is denoted by $\operatorname{Spec}(R)$. A $k$-ideal $A$ of $R$ is said to be $k$-prime or subtractive prime if it is a prime ideal of $R$. The family of all $k$-prime ideals of $R$ is denoted by $\operatorname{Spec}_{k}(R)$.

Lemma $2.2([12,15])$. If $R$ is a commutative semiring with zero and identity, then $\emptyset \neq$ $\operatorname{Max}_{k}(R) \subseteq \operatorname{Spec}_{k}(R)$.

A non-zero element $f$ in a commutative semiring $R$ with identity 1 is called a semiunit in $R$ if $1+r f=s f$ for some $r, s \in R$ [1], which is equivalent to the condition that $\overline{R f}=R$.

For an ideal $A$ of a semiring $R$, the set

$$
\sqrt{A}=\left\{a \in R \mid a^{n} \in A \text { for some } n \in \mathbb{N}\right\}
$$

is called the radical of $A$. Especially, if $R$ has zero 0 , then $\operatorname{Nil}(R)=\sqrt{\{0\}}$ is called the nilradical of $R$ [5].

Let $R$ be a commutative semiring with zero and identity. For each nonempty subset $S$ of $R$, put $U(S)=\{P \in \operatorname{Spec}(R) \mid S \subseteq P\}$. Then the collection $\{U(S) \mid \emptyset \neq S \subseteq R\}$ satisfies all the axioms of closed sets on a topology. The resulting topology is called the Zariski topology on $\operatorname{Spec}(R)$ and the topological space $\operatorname{Spec}(R)$ is called the prime spectrum of $R$ [5].

\section{3. $k$-Congruences on semirings}

In this section, for any semiring $R$, we introduce the $k$-congruences, establish criteria for $k$-congruences, show that there is an inclusion-preserving bijection between the $k$ congruences and $k$-ideals, and present an equivalent condition for the existence of a zero with the help of $k$-congruences.

Lemma 3.1. If $\theta$ is a congruence on $R$ and $R / \theta$ has a zero $O$, then $O$ is a k-ideal of $R$.

Proof. Show that $O$ is an ideal of $R$. If $a, b \in O$, then $[a+b]=[a]+[b]=O+O=O$ and so $a+b \in O$. If $a \in O$ and $r \in R$, then $[a r]=[a] \cdot[r]=O \cdot[r]=O$ and similarly $[r a]=O$, thus ar, $r a \in O$. Show that $O$ is $k$-closed in $R$. If $x+b \in O$ and $b \in O$, then $[x]=[x]+O=[x]+[b]=[x+b]=O$ and so $x \in O$.

Definition 3.2. A congruence $\theta$ on $R$ is called a subtractive congruence or $k$-congruence if there is an ideal $A$ of $R$ such that $\theta=\kappa_{A}$.

Denote by $\mathcal{K}(R)$ the family of all $k$-congruences on $R$.

Theorem 3.3. If $\theta$ is a congruence on $R$, then the following conditions are equivalent.

(1) $\theta$ is a k-congruence.

(2) $R / \theta$ has a zero $O$ and $\theta \subseteq \kappa_{O}$.

(3) $R / \theta$ has a zero $O$ and $\theta=\kappa_{O}$.

Proof. (1) $\Rightarrow(2)$ Suppose that $\theta=\kappa_{A}$ for an ideal $A$ of $R$. Then the $k$-closure $\bar{A}$ is a zero of $R / \theta$. If $x \theta y$, then $x+a=y+b$ for some $a, b \in A$ while $A \subseteq \bar{A}$ and so $x \kappa_{\bar{A}} y$.

$(2) \Rightarrow(3)$ By Lemma 3.1, $O$ is an ideal of $R$. Show that $\kappa_{O} \subseteq \theta$. If $x \kappa_{O} y$, then $x+a=y+b$ for some $a, b \in O$ and $[x]=[x]+O=[x]+[a]=[x+a]=[y+b]=[y]+[b]=$ $[y]+O=[y]$, thus $x \theta y$. Hence $\theta=\kappa_{O}$. 
$(3) \Rightarrow(1)$ This follows from Lemma 3.1.

Example 3.4. Let $R=\{0, a, b, c, d, 1\}$ be the lattice with the Hasse graph shown in Figure 3.1, which is not a distributive lattice. Define a multiplication $\cdot$ on $R$ by

$$
x \cdot y=\left\{\begin{array}{lc}
d, & \text { if } x, y \in\{1, b, c, d\} \\
0, & \text { otherwise }
\end{array}\right.
$$

Then $(R, \vee, \cdot)$ is a semiring and $A=\{0, a\}$ is an ideal of $(R, \vee, \cdot)$. Thus $\kappa_{A}$ is a $k$-congruence on $(R, \vee, \cdot)$ with congruence classes $\{0, a\}$ and $\{1, b, c, d\}$.

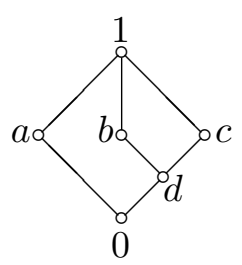

Figure 3.1

Example 3.5. Let $R=\{0, a, b, c, 1\}$ be the distributive lattice with the Hasse graph shown in Figure 3.2. Then the congruence $\theta$ on $(R, \vee, \wedge)$ with congruence classes $\{0\}$ and $\{1, a, b, c\}$ is not a $k$-congruence. In fact, the zero of $R / \theta$ is $O=\{0\}$ and $a \theta b$ while $a \vee 0 \neq b \vee 0$, which means that $\theta \nsubseteq \kappa_{O}$.<smiles>CC1C(=O)OC1=O</smiles>

Figure 3.2

By Lemma 3.1 and Theorem 3.3, we can define a function $\iota: \mathcal{K C}(R) \rightarrow \mathcal{K J}(R)$ by $\iota(\theta)=O_{R / \theta}$, where $O_{R / \theta}$ is a zero of $R / \theta$. Also we define a function $\kappa: \mathcal{J}(R) \rightarrow \mathcal{C}(R)$ by $\kappa(A)=\kappa_{A}$.

Then we obtain the following conclusion from Theorem 3.3.

Lemma 3.6. If $\theta$ is a k-congruence on $R$, then $\kappa(\iota(\theta))=\theta$.

Lemma 3.7. An ideal $A$ of $R$ is a $k$-ideal of $R$ iff $\iota(\kappa(A))=A$.

Proof. This follows from the fact that $\iota(\kappa(A))=\iota\left(\kappa_{A}\right)=O_{R / \kappa_{A}}=\bar{A}$.

Theorem 3.8. (1) The restriction of $\kappa$ to $\mathcal{K J}(R)$ is an inclusion-preserving bijection from $\mathcal{K J}(R)$ onto $\mathcal{K C}(R)$.

(2) If $\mathcal{K J}(R) \subseteq \mathcal{F} \subseteq \mathcal{J}(R)$ and $\kappa$ is injective on $\mathcal{F}$, then $\mathcal{F}=\mathcal{K J}(R)$.

Proof. (1) This follows from Lemmas 3.6 and 3.7.

(2) If $A \in \mathcal{F}$, then $\bar{A} \in \mathcal{K J}(R) \subseteq \mathcal{F}$. Since $\kappa(A)=\kappa_{A}=\kappa_{\bar{A}}=\kappa(\bar{A})$ and $\kappa$ is injective on $\mathcal{F}, A=\bar{A}$ and thus $A \in \mathcal{K J}(R)$.

Remark 3.9. Let $R$ be an additively idempotent semiring, i.e. $r+r=r$ for all $r \in R$. If $A$ is an ideal of $R$ and $x, y \in R$, then the condition $x+a=y+b$ for some $a, b \in A$ implies that $x+c=y+c$ for some $c \in A$. In fact, putting $c=a+b \in A$ gives $x+c=x+a+b=x+a+a+b=y+b+a+b=y+a+b=y+c$.

Remark 3.10. A characteristic one semiring is a commutative semiring with identity 1 such that $1+1=1[10,12]$. Obviously, characteristic one semirings are additively idempotent. 
Lescot [10] showed the existence of a bijection between the saturated ideals and excellent congruences in a characteristic one semiring. Actually, the saturated ideals are $k$-ideals and the excellent congruences are $k$-congruences. And Zhou and Yao [20] proved the existence of a one-to-one correspondence between the ideals which are lower sets and regular congruences in any additively idempotent semiring. By Han [6], the ideals which are lower sets are just $k$-ideals and every regular congruence is a $k$-congruence by Remark 3.9. Hence Theorem 3.8(1) is a generalization of Theorem 3.8 in [10] and Theorem 5 in [20].

Remark 3.11. $R \times R$ is always a $k$-congruence on $R$ because $\kappa_{R}=R \times R$. But id is not necessarily a $k$-congruence on $R$.

Theorem 3.12. $R$ has a zero 0 iff $\mathrm{id}_{R}$ is a $k$-congruence on $R$. In this case, $\kappa_{\{0\}}=\mathrm{id}_{R}$.

Proof. $(\Rightarrow)$ If $R$ has a zero 0 , then $\{0\}$ is an ideal of $R$ and obviously $\kappa_{\{0\}}=\operatorname{id}_{R}$.

$(\Leftarrow)$ If $\operatorname{id}_{R}$ is a $k$-congruence on $R$, then each of the congruence classes is a singleton. By Theorem 3.3, $R / \operatorname{id}_{R}$ has a zero $[a]=\{a\}$, where $a \in R$. Show that $a$ is a zero of $R$. For any $x \in R,[x+a]=[x]+[a]=[x]$ and so $x+a=x$. Also $[x a]=[x] \cdot[a]=[a]$ and similarly $[a x]=[a]$, thus $x a=a=a x$.

Theorem 3.12 enables us to give the following definition.

Definition 3.13. $R$ is said to be $k$-congruence-simple if it has no $k$-congruences other than $R \times R$ and id $R$.

$R$ is said to be $k$-simple or simple if it has no nontrivial $k$-ideals $[3,16] . R$ is said to be congruence-simple if it has just two congruences [4]. $R$ is said to be ideal-free if it has no nontrivial ideals [16]. Obviously, every congruence-simple semiring is $k$-congruence-simple and every ideal-free semiring is $k$-simple.

Theorem 3.14. $R$ is $k$-simple iff it is $k$-congruence-simple.

Proof. By Remark 3.11, $\kappa(R)=\kappa_{R}=R \times R$. By Theorem 3.12, $\kappa(\{0\})=\kappa_{\{0\}}=\mathrm{id}_{R}$ if $0 \in R$. Hence nontrivial $k$-ideals of $R$ bijectively correspond to $k$-congruences other than $R \times R$ and $\operatorname{id}_{R}$ under $\kappa$ by Theorem 3.8(1). This completes the proof.

Corollary 3.15. If $R$ is a ring, then the following statements are equivalent.

(1) $R$ is ideal-simple as a ring.

(2) $R$ is ideal-free as a semiring.

(3) $R$ is $k$-simple as a semiring.

(4) $R$ is congruence-simple as a ring.

(5) $R$ is congruence-simple as a semiring.

(6) $R$ is $k$-congruence-simple as a semiring.

Proof. Proposition 1.2 in [4] shows that (1), (2), (4) and (5) are equivalent. For any ring, a semiring ideal is a ring ideal iff it is a $k$-ideal. Thus (1) and (3) are equivalent. Hence Theorem 3.14 completes the proof.

\section{4. $k$-Prime spectrum}

In this section, for any commutative semiring $R$ with zero and identity, we prove that the $k$-prime spectrum is a compact subspace of the prime spectrum, and give a necessary and sufficient condition for the $k$-prime spectrum to be a $T_{1}$-space as well as necessary and sufficient conditions for the $k$-prime spectrum and its subsets to be irreducible. Finally, we prove that the $k$-prime spectrum is a spectral space.

Below, $R$ denotes any commutative semiring with zero 0 and identity 1 , and $X=$ $\operatorname{Spec}_{k}(R)$. Lemma 2.2 shows that $X \neq \emptyset$.

If $A \in \mathcal{J}(R)$, then $\sqrt{A}=\bigcap_{A \subseteq P \in \operatorname{Spec}(R)} P$ (see [5]). The following lemma gives a $k$-version of the radical of a $k$-ideal. 
Lemma 4.1. Let $X=\operatorname{Spec}_{k}(R)$. If $A \in \mathcal{K J}(R)$, then $\sqrt{A}=\bigcap_{A \subseteq P \in X} P$.

Proof. Show $\sqrt{A} \subseteq \bigcap_{A \subseteq P \in X} P$. If $a \in \sqrt{A}$, then $a^{n} \in A$ for some $n \in \mathbb{N}$. For every $k$-prime ideal $P$ with $A \subseteq P, a^{n} \in P$ and so $a \in P$. Hence $a \in \bigcap_{A \subseteq P \in X} P$.

Show the reverse inclusion. If $a \notin \sqrt{A}$, then $a^{n} \notin A$ for all $n \in \mathbb{N}$. Let $\Sigma$ be the family of all such $B \in \mathcal{K J}(R)$ that $A \subseteq B$ and $a^{n} \notin B$ for all $n \in \mathbb{N}$. Then $A \in \Sigma$ and $\Sigma$ is a poset under set-inclusion. For each chain $\left\{B_{\alpha} \mid \alpha \in \Lambda\right\}$ in $\Sigma, \bigcup_{\alpha \in \Lambda} B_{\alpha}$ is an upper bound of the chain in $\Sigma$. By Zorn's lemma, $\Sigma$ has a maximal element $Q$. Now show that $Q$ is a prime ideal. In fact, if $x y \in Q$ for some $x, y \in R \backslash Q$, then the maximality of $Q$ implies that $\overline{Q+R x} \notin \Sigma$ and $\overline{Q+R y} \notin \Sigma$. Thus $a^{m} \in \overline{Q+R x}$ and $a^{n} \in \overline{Q+R y}$ for some $m, n \in \mathbb{N}$. There are $p_{1}, p_{2}, q_{1}, q_{2} \in Q$ and $r_{1}, r_{2}, s_{1}, s_{2} \in R$ such that

$$
\begin{aligned}
a^{m}+p_{1}+r_{1} x & =p_{2}+r_{2} x, \\
a^{n}+q_{1}+s_{1} y & =q_{2}+s_{2} y .
\end{aligned}
$$

Multiplying two sides of Eq. 4.1 by $y$ gives

$$
a^{m} y+p_{1} y+r_{1} x y=p_{2} y+r_{2} x y
$$

and $a^{m} y \in Q$ since $x y \in Q$. Multiplying two sides of Eq. 4.2 by $a^{m}$ gives

$$
a^{m+n}+q_{1} a^{m}+s_{1} a^{m} y=q_{2} a^{m}+s_{2} a^{m} y,
$$

and so $a^{m+n} \in Q$, which contradicts $Q \in \Sigma$. Hence $Q \in X$. Meanwhile, since $Q \in \Sigma$, $a \notin Q$ and thus $a \notin \bigcap_{A \subseteq P \in X} P$.

Lemma 4.1 is a generalization of Theorem 5.1 and Proposition 5.5 in [11].

Corollary $4.2([12])$. For $A \in \mathcal{K J}(R), A=\sqrt{A}$ iff $A$ is an intersection of some $k$-prime ideals in $R$.

The following example shows that the assumption " $A$ is a $k$-ideal" cannot be omitted in Lemma 4.1.

Example 4.3. Let $B_{1}$ be the 2-element Boolean lattice and $R=B_{1}[x]$ be the polynomial semiring in the indeterminate $x$ over $B_{1}$. Consider the ideal

$$
A=\left\langle x^{2}+x, x^{3}+x\right\rangle=\left\{\left(x^{2}+x\right) f(x)+\left(x^{3}+x\right) g(x) \mid f, g \in R\right\}
$$

in $R$. Obviously, $x^{n} \notin A$ for all $n \in \mathbb{N}$ and so $x \notin \sqrt{A}$. Since $x+\left(x^{2}+x\right)=x^{2}+x, x \in \bar{A}$ and thus $A$ is not a $k$-ideal. If $P$ is a $k$-prime ideal of $R$ and $A \subseteq P$, then $\bar{A} \subseteq P$ and so $x \in P$. Hence $x \in \bigcap_{A \subseteq P \in \operatorname{Spec}_{k}(R)} P$.

For each nonempty subset $S$ of $R$, let $V(S)=\{P \in X \mid S \subseteq P\}$.

Lemma 4.4. (1) $V(\{0\})=X$ and $V(R)=\emptyset$.

(2) If $\emptyset \neq S \subseteq T \subseteq R$, then $V(T) \subseteq V(S)$.

(3) If $\emptyset \neq S \subseteq R$, then $V(S)=V(\langle S\rangle)=V(\overline{\langle S\rangle})$.

(4) If $A \in \mathcal{J}(R)$, then $V(A)=V(\sqrt{A})$.

(5) If $A, B \in \mathcal{J}(R)$, then $V(A) \cup V(B)=V(A B)=V(A \cap B)$.

(6) If $\left\{A_{i} \mid i \in \Lambda\right\} \subseteq \mathcal{J}(R)$, then $V\left(\sum_{i \in \Lambda} A_{i}\right)=\bigcap_{i \in \Lambda} V\left(A_{i}\right)$.

(7) If $A \in \mathcal{K J}(R), B \in \mathcal{J}(R)$ and $V(A) \subseteq V(B)$, then $B \subseteq \sqrt{A}$.

(8) For $A, B \in \mathcal{K J}(R), V(A)=V(B)$ iff $\sqrt{A}=\sqrt{B}$.

Proof. (1) through (3) are obvious. (4) through (6) are verified similarly to the case of $\operatorname{Spec}(R)$.

(7) If $A \subseteq P \in X$, then $P \in V(A) \subseteq V(B)$ and so $B \subseteq P$. Thus $B \subseteq \bigcap_{A \subseteq P \in X} P$. Lemma 4.1 shows that $B \subseteq \sqrt{A}$.

(8) $(\Rightarrow)$ follows from Lemma 4.1 .

$(\Leftarrow)$ follows from $(4)$. 
By Lemma 4.4(1)(3)(5)(6), the collection $\{V(S) \mid \emptyset \neq S \subseteq R\}$ satisfies all the axioms of closed sets on a topology. The resulting topology is called the Zariski topology and the topological space $X=\operatorname{Spec}_{k}(R)$ is called the $k$-prime spectrum of $R$. In fact, the $k$-prime spectrum $X=\operatorname{Spec}_{k}(R)$ is a subspace of the prime $\operatorname{spectrum~} \operatorname{Spec}(R)$.

For each nonempty subset $S$ of $R$, denote by $X_{S}$ the open subset $X \backslash V(S)$ of $X$. Then $X_{S}=\{P \in X \mid S \nsubseteq P\}$. In particular, for $a \in R, X_{\{a\}}$ is denoted simply by $X_{a}$ and called a basic open subset of $X$. Lemma 4.4(3) shows that $V(\langle a\rangle)=V(\{a\})=X \backslash X_{a}$. Obviously, $X_{S}=\bigcup_{a \in S} X_{a}$ and so we obtain the following conclusion.

Lemma 4.5. The collection $\left\{X_{a} \mid a \in R\right\}$ of all basic open subsets is a basis for open subsets for the Zariski topology on $X=\operatorname{Spec}_{k}(R)$.

Lemma 4.5 is a generalization of Proposition 6.2(2) in [11].

Theorem 4.6. For each $a \in R, X_{a}$ is a compact subset of $X=\operatorname{Spec}_{k}(R)$. Especially, $X=X_{1}$ is a compact topological space.

Proof. By Lemma 4.5, it suffices to verify that every covering of $X_{a}$ consisting of basic open subsets has a finite subcovering.

Let $X_{a} \subseteq \bigcup_{i \in \Lambda} X_{a_{i}}$ and $A=\overline{\left\langle\left\{a_{i} \mid i \in \Lambda\right\}\right\rangle}$. Lemma 4.4(3)(6) shows that

$$
V(\langle a\rangle) \supseteq \bigcap_{i \in \Lambda} V\left(\left\langle a_{i}\right\rangle\right)=V\left(\sum_{i \in \Lambda}\left\langle a_{i}\right\rangle\right)=V\left(\overline{\sum_{i \in \Lambda}\left\langle a_{i}\right\rangle}\right)=V(A),
$$

so $\langle a\rangle \subseteq \sqrt{A}$ by Lemma 4.4(7) and hence $a \in \sqrt{A}$. Then $a^{l} \in A$ for some $l \in \mathbb{N}$ and again

$$
a^{l}+t_{1} a_{i_{1}}+\cdots+t_{m} a_{i_{m}}=t_{m+1} a_{i_{m+1}}+\cdots+t_{n} a_{i_{n}}
$$

for some $i_{1}, \ldots, i_{m}, i_{m+1}, \ldots, i_{n} \in \Lambda$ and some $\left\{t_{i} \mid 1 \leq i \leq n\right\} \subseteq R$. Hence $a \in$ $\sqrt{\overline{\left\langle a_{i_{1}}, \ldots, a_{i_{n}}\right\rangle}}$. By Lemma 4.4(3)(4)(6),

$$
\begin{aligned}
V(\langle a\rangle) & \supseteq V\left(\sqrt{\overline{\left\langle a_{i_{1}}, \ldots, a_{i_{n}}\right\rangle}}\right)=V\left(\overline{\left\langle a_{i_{1}}, \ldots, a_{i_{n}}\right\rangle}\right) \\
& =V\left(\left\langle a_{i_{1}}, \ldots, a_{i_{n}}\right\rangle\right)=\bigcap_{k=1}^{n} V\left(\left\langle a_{i_{k}}\right\rangle\right)
\end{aligned}
$$

and so $X_{a} \subseteq \bigcup_{k=1}^{n} X_{a_{i_{k}}}$.

Theorem 4.6 is a generalization of Theorem 6.1 and Proposition 6.2(1) in [11].

Corollary 4.7. An open subset of $X=\operatorname{Spec}_{k}(R)$ is compact iff it is a union of a finite number of basic open subsets.

Remark 4.8. By Theorem 7.20 in [5], the prime spectrum $\operatorname{Spec}(R)$ is a $T_{0}$-space. Since $\operatorname{Spec}_{k}(R)$ is a $\operatorname{subspace} \operatorname{Spec}(R)$, it is also a $T_{0}$-space. However, $X=\operatorname{Spec}_{k}(R)$ is not necessarily a $T_{1}$-space.

The following theorem characterizes a semiring for which the $k$-prime spectrum is a $T_{1}$-space.

Theorem 4.9. $X=\operatorname{Spec}_{k}(R)$ is a $T_{1}$-space iff $k$-prime ideals are all $k$-maximal ideals in $R$.

Proof. $(\Rightarrow)$ Suppose that $X$ is a $T_{1}$-space and let $P$ be any $k$-prime ideal in $R$. A singleton subset $\{P\}$ is closed in $X$ and so $\{P\}=V(S)$ for some nonempty subset $S$ of $R$. Since $P \neq R$, Lemma 2.1 says that $P \subseteq M$ for some $k$-maximal ideal $M$. Since $M$ is a $k$-prime ideal by Lemma $2.2, M \in V(S)$ and so $P=M$, a $k$-maximal ideal.

$(\Leftarrow)$ For any $P \in X, P$ is a $k$-maximal ideal, so $\{P\}=V(P)$, a closed subset of $X$. Hence $X$ is a $T_{1}$-space. 
Example 4.10. For the semiring $\mathbb{Z}_{0}^{+}$of all nonnegative integers together with the usual addition and multiplication, the $k$-prime spectrum $\operatorname{Spec}_{k}\left(\mathbb{Z}_{0}^{+}\right)$consists of $\{0\}$ and all possible $\langle p\rangle$, where $p$ are primes, but it is not a $T_{1}$-space since the $k$-prime ideal $\{0\}$ is not a $k$-maximal ideal.

Example 4.11. For the 2-element Boolean lattice $B_{1}=\{0,1\},\{0\}$ is the unique $k$-prime ideal, thus the $k$-prime spectrum is $\operatorname{Spec}_{k}\left(B_{1}\right)=\{\{0\}\}$ and so it is a $T_{1}$-space.

A nonempty topological space $Z$ is said to be irreducible if every pair of nonempty open subsets in $Z$ has a nonempty intersection. A subset $Y$ of a topological space $Z$ is said to be irreducible if the subspace $Y$ is irreducible. A subset $Y$ of a topological space $Z$ is irreducible iff for any closed subsets $Y_{1}$ and $Y_{2}$ in $Z, Y \subseteq Y_{1} \cup Y_{2}$ implies either $Y \subseteq Y_{1}$ or $Y \subseteq Y_{2}[2]$.

Lemma 4.12. (1) If $f, e \in R$, then $X_{f} \cap X_{e}=X_{f e}$.

(2) $f \in \operatorname{Nil}(R)$ iff $X_{f}=\emptyset$.

(3) $X_{f}=X$ iff $f$ is a semiunit in $R$.

Proof. (1) If $P \in X_{f} \cap X_{e}$, then $f \notin P$ and $e \notin P$. Thus $f e \notin P$ and so $P \in X_{f e}$. Hence $X_{f} \cap X_{e} \subseteq X_{f e}$. The reverse inclusion is verified similarly.

(2) Lemma 4.1 shows that $\operatorname{Nil}(R)=\bigcap_{P \in X} P$. If $f \in \operatorname{Nil}(R)$, then for any $P \in X, f \in P$ and thus $X_{f}=\emptyset$. The converse is proved similarly.

(3) Let $f$ be a semiunit in $R$. If there is a $P \in X$ such that $P \notin X_{f}$, then $f \in P$ and $\overline{R f} \subseteq \bar{P}=P \neq R$, so $\overline{R f} \neq R$, which contradicts that $f$ is a semiunit. Hence $X \subseteq X_{f}$ and consequently $X=X_{f}$.

Conversely, suppose that $X_{f}=X$. Then for any $P \in X, P \in X_{f}$ and $f \notin P$. If $f$ is not a semiunit in $R$, then $\overline{R f} \neq R$, so by Lemma 2.1 , there is a $k$-maximal ideal $Q$ of $R$ such that $\overline{R f} \subseteq Q$. By Lemma 2.2, $Q \in X$ and so $Q \in X_{f}$, i.e. $f \notin Q$, which contradicts that $f \in \overline{R f} \subseteq Q$. Therefore, $f$ is a semiunit.

Lemma 4.12 is a generalization of Theorem 2.12 in [1].

Theorem 4.13. $X=\operatorname{Spec}_{k}(R)$ is irreducible iff $\mathrm{Nil}(R)$ is a k-prime ideal in $R$.

Proof. $(\Rightarrow)$ Suppose that $X$ is irreducible. By Lemma $4.1, \operatorname{Nil}(R)$ is a proper $k$-ideal in $R$. If $a, b \in R$ and $a b \in \operatorname{Nil}(R)$, then $X_{a b}=\emptyset$ by Lemma $4.12(2)$. Again, $X_{a} \cap X_{b}=\emptyset$ by Lemma 4.12(1). Since $X$ is irreducible, either $X_{a}=\emptyset$ or $X_{b}=\emptyset$, i.e. either $a \in \operatorname{Nil}(R)$ or $b \in \operatorname{Nil}(R)$. Hence $\operatorname{Nil}(R)$ is prime.

$(\Leftarrow)$ By Lemma 4.5 , it suffices to show that every pair of nonempty basic open subsets has a nonempty intersection. If $X_{a} \cap X_{b}=\emptyset$, then $X_{a b}=\emptyset$ by Lemma 4.12(1), and so $a b \in \operatorname{Nil}(R)$ by Lemma $4.12(2)$. By assumption, $\operatorname{Nil}(R)$ is a prime ideal, thus either $a \in \operatorname{Nil}(R)$ or $b \in \operatorname{Nil}(R)$. Again either $X_{a}=\emptyset$ or $X_{b}=\emptyset$ by Lemma $4.12(2)$. Hence $X$ is irreducible.

For $P \in X$, Lemma 4.4(3) shows that $\overline{\{P\}}=\bigcap_{P \in V(A)} V(A)$, where $A$ are ideals in $R$.

For each nonempty subset $Y$ of $X$, put $\tau(Y)=\bigcap_{P \in Y} P$. Then $\tau(Y)$ is a proper $k$-ideal of $R$.

Theorem 4.14. A nonempty subset $Y$ of $X=\operatorname{Spec}_{k}(R)$ is irreducible iff $\tau(Y)$ is a $k$ prime ideal of $R$.

Proof. $(\Rightarrow)$ Suppose that $Y$ is irreducible. If $a b \in \tau(Y)$, then $Y \subseteq V(\{a b\})$. By Lemma 4.4(3)(5), $V(\{a b\})=V(\langle a b\rangle)=V(\langle a\rangle\langle b\rangle)=V(\langle a\rangle) \cup V(\langle b\rangle)$. Thus $Y \subseteq V(\langle a\rangle) \cup V(\langle b\rangle)$. Since $Y$ is irreducible, either $V(\langle a\rangle) \supseteq Y$ or $V(\langle b\rangle) \supseteq Y$, and so either $a \in \tau(Y)$ or $b \in \tau(Y)$.

$(\Leftarrow)$ If $Y_{1}$ and $Y_{2}$ are closed subsets in $X$ with $Y \subseteq Y_{1} \cup Y_{2}$, then $Y_{1}=V\left(A_{1}\right)$ and $Y_{2}=$ $V\left(A_{2}\right)$ for some ideals $A_{1}$ and $A_{2}$ in $R$. By Lemma 4.4(5), $Y \subseteq V\left(A_{1}\right) \cup V\left(A_{2}\right)=V\left(A_{1} A_{2}\right)$ 
and so $A_{1} A_{2} \subseteq \tau(Y)$. Since $\tau(Y)$ is a prime ideal, either $A_{1} \subseteq \tau(Y)$ or $A_{2} \subseteq \tau(Y)$, i.e. either $Y \subseteq V\left(A_{1}\right)=Y_{1}$ or $Y \subseteq V\left(A_{2}\right)=Y_{2}$.

Corollary 4.15. For each $P \in X=\operatorname{Spec}_{k}(R), V(P)$ is irreducible.

Proof. In Theorem 4.14, putting $Y=V(P)$ gives $\tau(Y)=\bigcap_{Q \in V(P)} Q=P$, thus the conclusion follows.

Lemma 4.16. If $P \in \operatorname{Spec}_{k}(R)$, then $\overline{\{P\}}=V(P)$.

Proof. Since $P \in V(P)$, we have $\overline{\{P\}} \subseteq V(P)$. If $Q \in V(P)$, then $P \subseteq Q$ and for any ideal $A$ with $P \in V(A), A \subseteq P \subseteq Q$ and so $Q \in V(A)$, thus $Q \in \bigcap_{P \in V(A)} V(A)$. Hence $V(P) \subseteq \overline{\{P\}}$.

Theorem 4.17. If $Y$ is an irreducible closed subset of $\operatorname{Spec}_{k}(R)$, then $Y=\overline{\{P\}}$ for some $P \in \operatorname{Spec}_{k}(R)$.

Proof. Since $Y$ is closed, $Y=V(S)$ for some nonempty subset $S$ of $R$. Since $Y$ is irreducible, $P=\tau(Y)$ is a $k$-prime ideal by Theorem 4.14 .

Show that $V(P)=Y$. In fact, $S \subseteq \tau(Y)=P$ and $V(S) \supseteq V(P)$ by Lemma 4.4(2), so $V(P) \subseteq Y$. If $Q \in Y$, then $P=\tau(Y) \subseteq Q$ and so $Q \in V(P)$, thus $Y \subseteq V(P)$.

Lemma 4.16 is applied to show that $Y=\overline{\{P\}}$.

Let $Z$ be a topological space. If $Y$ is a closed subset of $Z$ and there exists a $y$ such that $Y=\overline{\{y\}}$, then $y$ is called a generic point of $Y . Z$ is called a spectral space if it is a compact $T_{0}$-space, the compact open subsets form a basis for the topology and are closed under finite intersection, and each of the irreducible closed subsets has a generic point [9].

Theorem 4.18. $\operatorname{Spec}_{k}(R)$ with the Zariski topology is a spectral space.

Proof. By Theorem 4.6, $\operatorname{Spec}_{k}(R)$ is a compact space. Remark 4.8 shows that $\operatorname{Spec}_{k}(R)$ is a $T_{0}$-space. By Lemma 4.5 and Theorem 4.6, the compact open subsets form a basis for the Zariski topology in $\operatorname{Spec}_{k}(R)$. By Corollary 4.7 and Lemma 4.12(1), the intersection of any finite number of compact open subsets is also a compact open subset in $\operatorname{Spec}_{k}(R)$. By Theorem 4.17, every irreducible closed subset has a generic point in $\operatorname{Spec}_{k}(R)$. Hence $\operatorname{Spec}_{k}(R)$ is a spectral space.

Theorem 4.18 generalizes the part about saturated prime ideals in Corollary 6.3 in [11].

\section{Zariski topology on $k$-prime congruences}

In this section, we prove that for any commutative semiring $R$ with zero 0 and identity 1 , the set of all the $k$-prime congruences with the Zariski topology is homeomorphic to the $k$-prime spectrum and so it is also a spectral space.

Definition 5.1. A congruence $\theta$ on a semiring $R$ is said to be prime if it satisfies the following two conditions:

(1) $\theta \neq R \times R$,

(2) $(a b) \theta 0$ implies either $a \theta 0$ or $b \theta 0$.

The collection of all $k$-prime congruences on $R$ is denoted by $\operatorname{CSpec}_{k}(R)$.

Lescot [10] defined the prime congruences in the setting of characteristic one semirings.

Lemma 5.2. (1) A congruence $\theta$ on $R$ is prime iff $O_{R / \theta}$ is a prime ideal in $R$.

(2) For any ideal $P$ in $R, \kappa_{P}$ is a prime congruence on $R$ iff $\bar{P}$ is a prime ideal in $R$.

Proof. (1) If $\theta$ is prime, then $\theta \neq R \times R$ and so $O_{R / \theta} \neq R$. If $a, b \in R$ and $a b \in O_{R / \theta}$, then $(a b) \theta 0$, so either $a \theta 0$ or $b \theta 0$. Hence either $a \in O_{R / \theta}$ or $b \in O_{R / \theta}$.

Conversely, if $O_{R / \theta}$ is a prime ideal, then $O_{R / \theta} \neq R$ and so $\theta \neq R \times R$. If $a, b \in R$ and ( $a b) \theta 0$, then $a b \in O_{R / \theta}$ and so either $a \in O_{R / \theta}$ or $b \in O_{R / \theta}$. Hence either $a \theta 0$ or $b \theta 0$.

(2) It follows from (1) since $O_{R / \theta}=\bar{P}$. 
For each nonempty subset $S$ of $R$, put

$$
W(S)=\left\{\theta \in \operatorname{CSpec}_{k}(R) \mid S \subseteq O_{R / \theta}\right\} .
$$

Lemma 5.3. (1) $W(\{0\})=\mathrm{CSpec}_{k}(R)$ and $W(R)=\emptyset$.

(2) If $\emptyset \neq S \subseteq T \subseteq R$, then $W(T) \subseteq W(S)$.

(3) If $\emptyset \neq S \subseteq R$, then $W(S)=W(\langle S\rangle)=W(\overline{\langle S\rangle})$.

(4) If $A \in \mathcal{J}(R)$, then $W(A)=W(\sqrt{A})$.

(5) If $A, B \in \mathcal{J}(R)$, then $W(A) \cup W(B)=W(A B)=W(A \cap B)$.

(6) If $\left\{A_{i} \mid i \in \Lambda\right\} \subseteq \mathcal{J}(R)$, then $\bigcap_{i \in \Lambda} W\left(A_{i}\right)=W\left(\bigcup_{i \in \Lambda} A_{i}\right)=W\left(\sum_{i \in \Lambda} A_{i}\right)$.

Proof. (1) Since $\{0\} \subseteq[0]=O_{R / \theta}$ for all $\theta \in \operatorname{CSpec}_{k}(R)$, the first equality holds. For any $\theta \in \operatorname{CSpec}_{k}(R), \theta \neq R \times R$ and so $O_{R / \theta} \neq R$. Thus the second equality holds.

(2) is obvious.

(3) Since $S \subseteq\langle S\rangle \subseteq \overline{\langle S\rangle}, W(\overline{\langle S\rangle}) \subseteq W(\langle S\rangle) \subseteq W(S)$ by (2). Now let $\theta \in W(S)$. Then $S \subseteq O_{R / \theta}$ and by Lemma $3.1, O_{R / \theta}$ is a $k$-ideal, so $\overline{\langle S\rangle} \subseteq O_{R / \theta}$. Thus $\theta \in W(\overline{\langle S\rangle})$.

(4) Since $A \subseteq \sqrt{A}, W(\sqrt{A}) \subseteq W(A)$ by (2). Now let $\theta \in W(A)$. Then $A \subseteq O_{R / \theta}$ and by Lemma 5.2(1), $O_{R / \theta}$ is a prime ideal of $R$ because $\theta$ is a prime congruence. Since $\sqrt{A}$ is the intersection of all prime ideals of $R$ containing $A$, we have $\sqrt{A} \subseteq O_{R / \theta}$. Thus $\theta \in W(\sqrt{A})$.

(5) Since $A B \subseteq A \cap B \subseteq A, W(A) \subseteq W(A \cap B) \subseteq W(A B)$ by (2). Similarly, $W(B) \subseteq$ $W(A \cap B) \subseteq W(A B)$ and so $W(A) \cup W(B) \subseteq W(A \cap B) \subseteq W(A B)$. Now let $\theta \in W(A B)$. Then $A B \subseteq O_{R / \theta}$ and by Lemma 5.2(1), $O_{R / \theta}$ is a prime ideal, so either $A \subseteq O_{R / \theta}$ or $B \subseteq O_{R / \theta}$. Thus either $\theta \in W(A)$ or $\theta \in W(B)$, i.e. $\theta \in W(A) \cup W(B)$.

(6) The first equality is obvious. Since $\left\langle\bigcup_{i \in \Lambda} A_{i}\right\rangle=\sum_{i \in \Lambda} A_{i}$, the second equality follows from (3).

Lemma 5.3(1)(3)(5)(6) shows that the family $\{W(S) \mid \emptyset \neq S \subseteq R\}$ satisfies all the axioms of closed sets on a topology. The resulting topology is called the Zariski topology on $\operatorname{CSpec}_{k}(R)$.

Theorem 5.4. $\operatorname{Spec}_{k}(R)$ is homeomorphic to $\operatorname{CSpec}_{k}(R)$ with respect to the Zariski topologies.

Proof. Define $\kappa: \operatorname{Spec}_{k}(R) \rightarrow \operatorname{CSpec}_{k}(R)$ by $\kappa(P)=\kappa_{P}$ and define $\iota: \operatorname{CSpec}_{k}(R) \rightarrow$ $\operatorname{Spec}_{k}(R)$ by $\iota(\theta)=O_{R / \theta}$. By Lemma 5.2 , both $\kappa$ and $\iota$ are well-defined, and

$$
\kappa \circ \iota=\operatorname{id}_{\mathrm{CSpec}_{k}(R)}, \quad \iota \circ \kappa=\operatorname{id}_{\operatorname{Spec}_{k}(R)} .
$$

We will show that $\kappa$ and $\iota$ are continuous. If $G$ is any closed $\operatorname{subset}$ in $\operatorname{CSpec}_{k}(R)$, then $G=W(S)$ for some nonempty subset $S$ of $R$. Since

$$
\begin{aligned}
P \in \kappa^{-1}(G) & \Leftrightarrow \kappa_{P}=\kappa(P) \in G=W(S) \\
& \Leftrightarrow S \subseteq O_{R / \kappa_{P}}=\bar{P}=P \Leftrightarrow P \in V(S),
\end{aligned}
$$

we have $\kappa^{-1}(G)=V(S)$, a closed subset in $\operatorname{Spec}_{k}(R)$. Hence $\kappa$ is continuous. If $H$ is any closed subset in $\operatorname{Spec}_{k}(R)$, then $H=V(S)$ for some nonempty subset $S$ of $R$. Since

$$
\begin{aligned}
\theta \in \iota^{-1}(H) & \Leftrightarrow O_{R / \theta}=\iota(\theta) \in H=V(S) \\
& \Leftrightarrow S \subseteq O_{R / \theta} \Leftrightarrow \theta \in W(S),
\end{aligned}
$$

we have $\iota^{-1}(H)=W(S)$, a closed subset in $\operatorname{CSpec}_{k}(R)$. Hence $\iota$ is continuous.

Therefore, $\kappa$ and $\iota$ are both homeomorphisms.

By Theorems 4.18 and 5.4, we draw the following conclusion.

Theorem 5.5. $\operatorname{CSpec}_{k}(R)$ with the Zariski topology is a spectral space. 
Acknowledgment. The author is grateful to the anonymous referees for their valuable suggestions that have improved the presentation of this paper significantly.

\section{References}

[1] S.E. Atani and R.E. Atani, A Zariski topology for k-semirings, Quasigroups Related Systems 20, 29-36, 2012.

[2] M.F. Atiyah and I.G. Macdonald, Introduction to Commutative Algebra, Addison Wesley, Massachusetts, 1969.

[3] F. Callialp, G. Ulucak and U. Tekir, On the Zariski topology over an L-module M, Turkish J. Math. 41, 326-336, 2017.

[4] R. El Bashir and T. Kepka, Congruence-simple semirings, Semigroup Forum 75, 588-608, 2007.

[5] J.S. Golan, Semirings and their Applications, Kluwer Academic Publishers, Dordrecht, 1999.

[6] S.C. Han, Maximal k-ideals and r-ideals in semirings, J. Algebra Appl. 14 (10), 1250195, 13 pages, 2015.

[7] U. Hebisch and H.J. Weinert, Semirings: Algebraic Theory and Applications in Computer Science, World Scientific, Singapore, 1998.

[8] M. Henriksen, Ideals in semirings with commutative addition, Amer. Math. Soc. Notices 5, 321, 1958.

[9] M. Hochster, Prime ideal structure in commutative rings, Trans. Amer. Math. Soc. 142, 43-60, 1969.

[10] P. Lescot, Absolute algebra II - Ideals and spectra, J. Pure Appl. Algebra 215, 17821790, 2011.

[11] P. Lescot, Absolute algebra III - The saturated spectrum, J. Pure Appl. Algebra 216, 1004-1015, 2012.

[12] P. Lescot, Prime and primary ideals in semirings, Osaka J. Math. 52, 721-736, 2015.

[13] G. Maze, C. Monico and J. Rosenthal, Public key cryptography based on semigroup actions, Adv. Math. Commun. 1, 489-507, 2007.

[14] A. Pena, L.M. Ruza and J. Vielma, Separation axioms and the prime spectrum of commutative semirings, Rev. Notas Mat. 5 (2), 66-82, 2009.

[15] M.K. Sen and M.R. Adhikari, On maximal k-ideals of semirings, Proc. Amer. Math. Soc. 118, 699-703, 1993.

[16] H.E. Stone, Matrix representation of simple halfrings, Trans. Amer. Math. Soc. 233, 339-353, 1977.

[17] H.S. Vandiver, Note on a simple type of algebra in which the cancellation law of addition does not hold, Bull. Amer. Math. Soc. 40, 916-920, 1934.

[18] H.J. Weinert, M.K. Sen and M.R. Adhikari, One-sided k-ideals and h-ideals in semirings, Math. Pannon. 7 (1), 147-162, 1996.

[19] G. Yesilot, On prime and maximal k-subsemimodules of semimodules, Hacet. J. Math. Stat. 39, 305-312, 2010.

[20] B. Zhou and W. Yao, Relations between ideals and regular congruences in idempotent semirings with a zero, Basic Sci. J. Textile Univ. 24, 253-255, 2011. 